\title{
Versión Breve del STAI en Adolescentes y Universitarios Españoles
}

\author{
STAI Short Version in Adolescents and College Spanish
}

\author{
Alejandro Guillén-Riquelme \\ Gualberto Buela-Casal \\ Universidad de Granada, España
}

(Rec: 3 de julio de 2013 / Acept:16 de agosto de 2013)

\begin{abstract}
Resumen
El STAI es uno de los instrumentos de evaluación psicológica más empleados, generándose diversas versiones breves del mismo. No se han hallado versiones breves en las que se haya comprobado el ajuste para muestras de adolescentes ni universitarios españoles. El objetivo de este artículo es comprobar cuál de las versiones cortas propuestas en la literatura tiene un mejor ajuste en adolescentes y universitarios. Para ello, se aplicó el STAI a 482 adolescentes escolarizados y 510 estudiantes universitarios, de diversas ciudades españolas. Mediante AFC se evaluó el ajuste de cinco versiones breves del STAI. En los universitarios hay dos modelos en los que se observa un buen ajuste. En los adolescentes no ajusta ningún modelo. Puede que el proceso de diferenciación emocional con la edad explique que en los adolescentes las versiones breves halladas (siempre con adultos) no serían válidas, sí pudiendo emplearse con universitarios.

Palabras clave: STAI, versión breve, ansiedad, adolescencia.
\end{abstract}

\begin{abstract}
The STAI is an instrument very used, generating different short versions. We found no short versions in which it is tests the fit for teenagers and college samples Spanish. The aim of this paper is to test which of the short versions proposed in the literature have a better fit in adolescents and college students. To do this, we applied the STAI to 482 adolescent students and 510 university students from different Spanish cities. Through AFC we evaluated five short versions of the STAI. At the university there are two models in which there is a good fit. In adolescents not fit any model. Maybe the emotional differentiation process explains that the found short versions (always with adults) are not valid in teenagers, and can be used with university students.
\end{abstract}

Key words: STAI, short version, anxiety, adolescence.

\section{Introducción}

El State-Trait Anxiety Inventory es uno de los instrumentos clásicos para evaluar ansiedad, uno de los trastornos psicológicos con una mayor prevalencia en población general (Beutel, Bleichner, von Heumann, Tritt y Hardt, 2011) así como en universitarios (Micin y Bagladi, 2011). Desarrollado por Spielberger, Gorsuch y Lushene (1970) este cuestionario ha sido citado en más de 14.000 estudios de archivo, siendo uno de los instrumentos más empleados por psicólogos (Spielberger y Reheiser, 2009). Su aplicación permite obtener dos escalas: una de ansiedad rasgo (tendencia estable de percibir estímulos como más amenazantes) y ansiedad estado (presencia de estímulos ansiosos en el entorno del evaluado en los días cercanos a la evaluación). Además, el STAI ha contado con más de 60 adaptaciones o traducciones (Spielberger y Reheiser, 2009), entre ellas, la española (Buela-Casal, Guillén-Riquelme y Seisdedos, 2011), donde es el sexto cuestionario más utilizado por psicólogos clínicos (Muñiz y Fernández-Hermida, 2010). Esta adaptación cuenta con adecuadas propiedades psicométricas en población general (Guillén-Riquelme y Buela-Casal, 2011). 
En 1983, se realiza una doble modificación del STAI: a) cambia la dirección de algunos ítems, para equiparar el número de elementos positivos y negativos; b) varía el contenido de otros, para facilitar su comprensión (Spielberger, 1983). Estos cambios dan respuesta a diversas críticas realizadas sobre el STAI. A la primera versión se le denomina como forma $\mathrm{X}$ y a la versión con los cambios descritos, como forma Y. Este hecho es importante, porque el contenido de algunos ítems varía según la versión (forma $\mathrm{X}$ o Y), pese a que las evaluaciones realizadas con una u otra no difieran (Spielberger, 1983). La adaptación española se realizó a partir de la forma X (Buela-Casal et al., 2011).

Diversos autores realizan una versión reducida de los ítems del cuestionario que permite evaluar ansiedad de forma fiable. La versión breve realizada por van Knippenberg, Duivenvoorden, Bonke y Passchier (1990), aplica, por primera vez, una metodología científica y unas pruebas estadísticas adecuadas; requisitos que no cumplen las propuestas anteriores. Estos autores realizan nueve versiones breves, a partir de las correlaciones ítem-total corregidas. Las versiones varían en el número de ítems (combinaciones de 4, 6 y 8 ítems). Además, utilizan dos muestras de pacientes médicos y una de estudiantes universitarios. En este trabajo, se concluye que es posible establecer versiones breves del STAI, con adecuadas propiedades psicométricas. También, se establece que las versiones de seis y ocho ítems son las adecuadas para la evaluación de la ansiedad estado y rasgo; mientras que las de cuatro ítems resultan insuficientes. En la muestra de universitarios, la versión breve está formada por los ítems número $2,4,8,11,12,15,17$ y 18 para la ansiedad estado, y por los ítems $7,8,14,15,16,17,18$ y 20 para la ansiedad rasgo. Hay que precisar que las versiones de 6 ítems se obtienen, eliminando, en la escala de ansiedad estado, los ítems 8 y 12 , y, en la de ansiedad rasgo, los ítems 8 y 20 .

Recientemente, se han propuesto nuevas versiones breves del STAI. Kaipper, Chachamovich, Hidalgo, Torres y Caumo (2010) realizaron una versión breve de ambas escalas. El banco de ítems estaba formado por todos los ítems de la forma $\mathrm{X}$, más los ítems con diferente contenido de la forma Y. Estos autores establecen que los ítems de la versión breve de la escala de ansiedad estado son: 1, 2, 5, 6, 7, 8, 11, 13, 14, 16, 17,18 y 19, además de otros ítems de la forma Y. En el caso de la ansiedad rasgo los ítems seleccionados son: 1, 2, 5, 7, 8, $9,10,12,16,17,18$ y 20, además de otros ítems de la forma Y. Esta versión breve tiene 25 ítems pero, en la actualidad, también se defienden versiones de seis ítems por cada una de las escalas. De esta forma, Fioravanti-Bastos, Cheniaux y Landeira-Fernandez (2011) afirman que la selección de los ítems 1, 3, 5, 12, 15 y 17, para la ansiedad estado y los ítems 7, 9, 11, 13, 16 y 20 para la ansiedad rasgo, resultan adecuados para evaluar estos factores.

En España se realizó una versión breve mediante una muestra de población general, empleando análisis discriminante (Buela-Casal y Guillén-Riquelme, en prensa). Esta versión está compuesta por los ítems 3, 8, 16, 18 y 20 para la ansiedad estado y los ítems $3,8,10,12$ y 18 para la ansiedad rasgo. Por otra parte, se comparaba, mediante análisis factorial confirmatorio, si el ajuste de esa versión corta era mejor que el de otras reducciones anteriores, concluyendo que la versión breve con un mejor ajuste era la presentada por van Knippenberg et al. (1990). Además del trabajo de Buela-Casal y Guillén-Riquelme (en prensa), se ha realizado otra versión breve con muestra española (Perpiñá-Galvañ, Richart-Martínez y Cabañero-Martínez, 2011; Perpiñá-Galvañ, Richart-Martínez, Cabañero-Martínez y Martínez-Durá, 2011). Sin embargo, esta versión muestra un par de problemas: a) únicamente emplea muestra de pacientes con intubación respiratoria; b) se realiza la confirmación de la versión propuesta por Marteau y Bekker (1992), derivada de la forma Y del STAI, cuyos ítems sólo coinciden parcialmente con los de la adaptación española, proveniente de la forma X (Buela-Casal et al., 2011).

El STAI se carateriza por la calidad de sus propiedades psicométricas a la hora de evaluar ansiedad en diversas poblaciones, entre ellas la de adolescentes. Así pues, la adaptación española resulta fiable y válida, presentando baremos específicos para este segmento de la población (Buela-Casal et al., 2011). Además, la adaptación chilena (desarrollada a partir de la versión española) también posee una consistencia interna elevada al ser aplicada en una muestra de adolescentes (Vera-Villarroel, Celis-Atenas, Córdova-Rubio, Buela-Casal y Spielberger, 2007).

Entre las numerosas versiones breves del STAI ninguna sobresale por un mayor apoyo empírico ni ha sido validada en población adolescente. Por ello, el objetivo del presente trabajo es comprobar si las diferentes versiones breves realizadas, a partir de la forma X del STAI, ajustan adecuadamente en: a) una muestra de adolescentes españoles y b) una muestra de universitarios. Al utilizar ambas muestras, se podrá comparar, de forma más precisa, el ajuste de la versión corta en la adolescencia y al inicio de la edad adulta. La hipótesis inicial es el adecuado ajuste de los diferentes modelos, siendo los hallados por van Knippenberg et al. (1990) y por Buela-Casal y Guillén-Riquelme (en prensa), los que ajusten de forma más precisa en adolescentes y universitarios, puesto que en ellos se ha observado mejores propiedades psicométricas en una muestra de adultos españoles. 


\section{Método}

\section{Participantes}

Para la consecución del objetivo planteado, se utilizaron dos muestras independientes. La primera de ellas formada por 482 adolescentes de diversos institutos (públicos y privados) de cuatro ciudades españolas. Del total, $186(42.2 \%)$ son varones. La media de edad es de $15.48(D T=1.58)$, siendo el mínimo de 12 y el máximo de 19. En el caso de los varones la media es de 15.31 años $(D T=1.76)$. En las mujeres la media es de 15.61 años $(D T=1.59)$.

La segunda muestra está formada por 510 universitarios matriculados en seis universidades españolas (todas ellas públicas), procedentes de diversas carreras. La media de edad es de 23.15 años $(D T=5.51)$ y el rango de edad abarca desde 18 a 54 años, con más de un $90 \%$ de los participantes menores de 29 años). En el caso de los varones ( $n=186$; $36.5 \%)$ la media de edad es de 25.05 años $(D T=7.71)$. En las mujeres la edad media es de 22.06 años $(D T=3.96)$.

\section{Instrumentos}

Tras realizar preguntas relativas al lugar de residencia, sexo y edad, se procedió a aplicar la adaptación española del State-Trait Anxiety Inventory (Buela-Casal et al., 2011). Este cuestionario permite evaluar ansiedad rasgo (tendencia a percibir los estímulos como ansiógenos o no) y estado (grado en el que hay elementos ansiosos en el entorno de la persona en el tiempo cercano a la evaluación). La escala de respuesta es de tipo Likert con 4 alternativas (en la adaptación española desde 0 hasta 3). Tanto la ansiedad estado como la ansiedad rasgo cuentan con 20 ítems para su evaluación. Por ello, el rango de respuestas va desde 0 a 60, correspondiéndose mayor nivel de ansiedad con una mayor puntuación. La adaptación española tiene adecuadas propiedades psicométricas (Guillén-Riquelme y Buela-Casal, 2011; Buela-Casal et al., 2011). Además, la escala se puede aplicar en población adolescente así como universitarios, teniendo evidencias de fiabilidad y validez.

\section{Procedimiento}

El procedimiento es diferente para cada una de las muestras obtenidas. En el caso de la muestra de adolescentes, en primer lugar se contactó con diversos centros de cuatro ciudades españolas. La aplicación de los cuadernillos transcurrió en la propia aula, durante las horas de tutorías a grupos completos (entre 25 y 35 alumnos de forma general). El experimentador informaba de la confidencialidad del estudio así como del consentimiento informado. Seguidamente se repartieron los cuadernillos al grupo y el experimentador permaneció en el aula durante la aplicación, para resolver posibles dudas de cumplimentación.

En el caso de la muestra de universitarios, se contactó con los centros para la colaboración. El experimentador se personó en las aulas e informó sobre la confidencialidad y el consentimiento informado. De nuevo se aplicó la prueba a grupos completos, pese a que en este caso eran, de media, tres veces más numerosos que los de los adolescentes. Durante la prueba, el experimentador permaneció en el aula para contestar posibles dudas.

\section{Análisis de datos}

El análisis factorial confirmatorio fue seleccionado como la técnica analítica idónea para el ajuste de los modelos. Esta aproximación permite facilitar la comparaciones de invarianza y equivalencia para futuros estudios, garantizando la utilización del test en diversas muestras (Muñiz, Elosua y Hambleton, 2013). Se pusieron a prueba cinco modelos diferentes: el modelo 1 se corresponde al presentado por Buela-Casal y Guillén-Riquelme (en prensa); el modelo 2 es el propuesto por van Knippenberg et al. (1990) de 6 ítems; el modelo 3 se corresponde con el anterior pero en la versión de 8 ítems; el modelo 4 es el propuesto por Kaipper et al. (2010) y el modelo 5 por Fioravanti-Bastos et al. (2011). La naturaleza categórica de los ítems (escala de cuatro alternativas) hizo necesario aplicar una prueba que solventase la falta de normalidad, resultante del uso de este tipo de variables. Así pues, se seleccionó el método robusto a partir del Weighted Least Squares (WLSMV; Brown, 2006). La comprobación del ajuste de los modelos a los datos se realizó mediante cuatro estadísticos: el $\chi^{2}$ dividido por los grados de libertad, el Comparative Fit Index (CFI), el Tucker-Lewis Index (TLI) y el Root Mean Square Error of Approximation (RMSEA). Los dos primeros deben tener valores superiores al 0.95 para que se considere que el ajuste es adecuado. En el RMSEA se exigen valores inferiores a 0.08 . A la hora de establecer el modelo se incluyeron, de forma conjunta, ambas escalas (estado y rasgo) y su covariación, ya que estudios previos indicaban una fuerte relación entre ellas (BuelaCasal y Guillén-Riquelme, en prensa). Para los análisis se utilizó el paquete lavaan (Rosseel, 2012) del programa $R$. Tras ello, la redacción del artículo se realizó siguiendo las recomendaciones propuestas por Hartley (2012). 


\section{Resultados}

\section{Análisis factorial confirmatorio en adolescentes}

En la muestra de adolescentes se comprobó el ajuste de los cinco modelos teóricos, expuestos anteriormente. En cada uno de ellos y, en función de los índices de modificación, se incluyeron diversas covarianzas de los errores, respetando que: 1) los errores fuesen de ítems de un mismo factor, 2) el índice de modificación tuviese un valor superior a 20 puntos. En el modelo número 4 al tener un mayor número de ítems, para evitar una sobrecarga de covariaciones de errores, se amplió este criterio hasta un valor de 30 puntos. En la Tabla 1, se recoge un resumen de los índices de ajuste de cada uno de los modelos teóricos. Ninguno de ellos alcanza niveles adecuados de ajuste, aunque, en el modelo 5 se observan valores más cercanos a un ajuste adecuado.

\section{Análisis factorial confirmatorio en universitarios}

En el caso de los universitarios, hay un correcto ajuste de diferentes modelos. El modelo 1 tiene índices próximos a los criterios establecidos. El modelo 2, conformado por 6 ítems, tiene un mejor ajuste que el resto, en la muestra de universitarios. El modelo 3 tiene un ajuste similar al anterior, siendo ligeramente inferior, y contando con dos ítems más por cada una de las escalas. El modelo 4 es, junto con el 1, el que cuenta con índices de ajuste más bajos. El modelo 5, no llega a ajustar adecuadamente, aunque el CFI resulte superior a 0.95 . Un resumen se recoge en la Tabla 2.

Tras analizar el ajuste se comprobó la covariación entre factores. En el modelo 2 (el que tiene mejores índices) la covariación es de 0.89 , lo que indica una fuerte relación entre ambos factores. En el resto de modelos también se encuentra valores similares entre la covariación de la ansiedad estado y rasgo.

\section{Análisis de fiabilidad en el modelo con mejor ajuste en universitarios}

A partir de los ítems del modelo que mejor ajuste obtiene, en la muestra de universitarios, se realizó un análisis de la fiabilidad para cada una de las escalas. En el caso de la ansiedad estado, el alfa de Cronbach es de 0.82. En la correlación ítem total corregida los valores oscilan desde 0.52 a 0.73 , por lo que todos los ítems están relacionados con el total. En el caso de la ansiedad rasgo el alfa de Cronbach es de 0.69 (la eliminación de un ítem mejoraría el alfa en dos centésimas). En la correlación ítem total corregida se observan resultados dispares ya que la correlación de los

Tabla 1. Ajuste de los modelos propuestos en el análisis factorial confirmatorio en adolescentes

\begin{tabular}{|c|c|c|c|c|c|}
\hline \multirow{2}{*}{ Estadístico } & \multicolumn{5}{|c|}{ Modelo } \\
\hline & $\mathrm{m} 1$ & $\mathrm{~m} 2$ & $\mathrm{~m} 3$ & $\mathrm{~m} 4$ & $\mathrm{~m} 5$ \\
\hline$\chi^{2}$ & 231.65 & 346.20 & 583.29 & $1,482.25$ & 230.51 \\
\hline Grados de libertad & 28 & 47 & 95 & 255 & 44 \\
\hline$\chi^{2 / \text { Grados de libertad }}$ & 8.27 & 7.37 & 6.14 & 5.81 & 5.24 \\
\hline $\mathrm{p}$ & $<0.001$ & $<0.001$ & $<0.001$ & $<0.001$ & $<0.001$ \\
\hline Comparative Fit Index $(C F I)$ & 0.887 & 0.906 & 0.889 & 0.936 & 0.891 \\
\hline Tucker-Lewis Index (TLI) & 0.818 & 0.868 & 0.86 & 0.925 & 0.837 \\
\hline Root Mean Square Error of Approximation (RMSEA) & 0.122 & 0.114 & 0.103 & 0.099 & 0.093 \\
\hline I.C. inf. RMSEA & 0.108 & 0.103 & 0.095 & 0.094 & 0.082 \\
\hline I.C. sup. RMSEA & 0.137 & 0.126 & 0.111 & 0.104 & 0.105 \\
\hline
\end{tabular}

Nota. Se empleó como método de ajuste el Weigthed Least Squares. m = modelo. El modelo 1: datos de Buela-Casal y GuillénRiquelme (en prensa); modelo 2: propuesto por van Knippenberg et al. (1990) de 6 ítems; modelo 3: propuesto por van Knippenberg et al. (1990) de 8 ítems, modelo 4: propuesto por Kaipper et al. (2010); modelo 5: Fioravanti-Bastos et al. (2011) modificado. $p=$ probabilidad; I.C. = intervalo de confianza al $95 \%$. 
ítems $15,16,17$ y 18 oscila de 0.42 a 0.55 , mientras en el ítem 7 , es de 0.27 y en el ítem 14 es de 0.38 .

\section{Discusión}

En el prolegómeno de este estudio se propuso como objetivo "comprobar si las diferentes versiones breves realizadas, a partir de la forma X del STAI, ajustan adecuadamente en: a) una muestra de adolescentes españoles y b) una muestra de universitarios", también se partió de la hipótesis de que se obtendría un adecuado ajuste de los modelos en ambas muestras. En los universitarios la escala breve que mejor ajuste ha mostrado, coincide con la establecida por Buela-Casal y Guillén-Riquelme (en prensa) en población general española. Se confirma que la versión breve propuesta para población general (BuelaCasal y Guillén-Riquelme, en prensa), a partir de la versión de seis ítems de van Knippenberg et al. (1990), se puede utilizar para evaluar ansiedad en universitarios. El hecho de contar con una versión breve de este cuestionario tan empleado resulta necesario, no solo en los grupos analizados, si no también en pacientes con problemas físicos y que requieran varias evaluaciones (Martínez-López, Durá Ferrandis, Andreu Vaillo, Galón Garrido, Murgui Pérez y Ibáñez Guerra, 2012).
Ninguna de las versiones breves del STAI, incluida la seleccionada para los universitarios, muestra un correcto ajuste en el grupo de adolescentes. La nula incidencia de un buen ajuste en el grupo de adolescentes, de las cinco versiones breves teóricas propuestas, resulta extraña ya que la versión completa del STAI tiene adecuadas propiedades psicométricas en esta población (Buela-Casal et al., 2011). El hecho de que en el grupo de adolescentes no se ajuste ninguna de las versiones breves teóricas tiene varias explicaciones. En primer lugar, cabe indicar que la mayoría de las versiones breves se realizaron a partir de muestras de adultos (con o sin trastornos de ansiedad). Por ello, puede que ninguna de estas versiones se ajuste a una muestra de adolescentes. Sin embargo, este argumento no justifica el hecho de que la versión corta para adultos no se pueda aplicar a adolescentes, mientras que en el STAI completo se observan adecuadas propiedades psicométricas en esta muestra (Buela-Casal et al., 2011; Vera-Villaroel et al., 2007). Una posible explicación a este hecho se puede hallar en la teoría general del afecto, donde se defiende que la evolución de las emociones se produce de un modo similar a la de la inteligencia, según la hipótesis de la diferenciación. Se considera que se parte de afectos muy generales, desde los cuales, a partir de un proceso de diferenciación, se desarrollan las emociones más específicas, permitiendo diferenciarlas entre sí. Así pues, Yik, Russell y Feldman-Barrett (1999) proponen la existencia

Tabla 2. Ajuste de los modelos propuestos en el análisis factorial confirmatorio en universitarios

\begin{tabular}{|c|c|c|c|c|c|}
\hline \multirow{2}{*}{ Estadístico } & \multicolumn{5}{|c|}{ Modelo } \\
\hline & $\mathrm{m} 1$ & $\mathrm{~m} 2$ & $\mathrm{~m} 3$ & $\mathrm{~m} 4$ & $\mathrm{~m} 5$ \\
\hline$\chi^{2}$ & 160.54 & 143.97 & 358.58 & $1,487.54$ & 202.18 \\
\hline Grados de libertad & 29 & 44 & 94 & 259 & 45 \\
\hline$\chi 2 /$ Grados de libertad & 5.54 & 3.27 & 3.81 & 5.74 & 4.49 \\
\hline $\mathrm{p}$ & $<0.001$ & $<0.001$ & $<0.001$ & $<0.001$ & $<0.001$ \\
\hline Comparative Fit Index $(C F I)$ & 0.942 & 0.976 & 0.96 & 0.964 & 0.959 \\
\hline Tucker-Lewis Index (TLI) & 0.909 & 0.964 & 0.949 & 0.959 & 0.939 \\
\hline Root Mean Square Error of Approximation (RMSEA) & 0.094 & 0.067 & 0.074 & 0.096 & 0.083 \\
\hline I.C. inf RMSEA & 0.08 & 0.055 & 0.066 & 0.092 & 0.071 \\
\hline I.C. sup RMSEA & 0.109 & 0.079 & 0.083 & 0.101 & 0.095 \\
\hline
\end{tabular}

Nota. Se empleó como método de ajuste el Weigthed Least Squares. $\mathrm{m}=$ modelo. El modelo 1: datos de este artículo; modelo 2: propuesto por van Knippenberg et al. (1990) de 6 ítems; modelo 3: propuesto por van Knippenberg et al. (1990) de 8 ítems, modelo 4: propuesto por Kaipper et al. (2010); modelo 5: Fioravanti-Bastos et al. (2011) modificado. $p=$ probabilidad; I.C. = intervalo de confianza al 95\%. 
de dos continuos en los que se enmarcarían las diferentes emociones: el primero de ellos el arousal, oscilante desde la activación a la desactivación; el segundo el de la valencia del afecto, indicativo del carácter positivo o negativo de los sentimientos. La ansiedad se vincularía exclusivamente con afecto negativo (Watson, Clark y Stasik, 2011). De acuerdo con este modelo, las emociones son más generales en la adolescencia que en el inicio de la edad adulta (tramo en el que se encuentra la muestra de universitarios), ya que la diferenciación se incrementa con el desarrollo psicológico del individuo. Por tanto, en adolescentes de muy tempranas edades puede ser recomendable emplear versiones breves de cuestionarios más generales sobre sensibilidad ansiosa (por ejemplo: Kämpfe et al., 2012).

En los universitarios el modelo que mejor ajusta es el presentado por van Knippenberg et al. (1990) lo que confirma la hipótesis inicial, donde se enunciaba que dicha versión tendría el mejor ajuste, puesto que esta propuesta fue la que se seleccionó como versión breve en población general española (Buela-Casal y Guillén-Riquelme, en prensa). Este hecho, confirma que se puede emplear una versión breve en universitarios, pese a que tengan mayores puntuaciones medias en ansiedad que la población general (Buela-Casal et al., 2011), pueden ser evaluados empleando una versión reducida de la misma. Además, al considerar el grupo de universitarios como una parte de la población general, se aporta una nueva evidencia de que la versión breve seleccionada en la investigación de Buela-Casal y Guillén-Riquelme (en prensa), es la más apropiada. Observando otras versiones breves de cuestionarios de ansiedad que ajustan bien en este tipo de muestras (por ejemplo: Antúnez y Vinet, 2012). Así pues, la versión de seis ítems de van Knippenberg et al. (1990) permite evaluar ansiedad estado y rasgo en población general (incluyendo al grupo de universitarios).

En resumen, la versión propuesta por van Knippenberg et al. (1990) y confirmada por Buela-Casal y Guillén-Riquelme (en prensa), compuesta por los ítems 2, 4, 11, 15, $17 \mathrm{y}$ 18, para la ansiedad estado y por los ítems $7,14,15,16$, 17 y 18 para la ansiedad rasgo, permite evaluar de forma fiable ambos factores en universitarios. En el caso de los adolescentes, pese a que la versión completa sea válida, las versiones breves utilizadas hasta la fecha no proporcionan un correcto ajuste. Es imprescindible hallar una versión breve que permita evaluar ansiedad en adolescentes de forma válida y fiable. Una de las limitaciones del presente estudio es que las muestras no están seleccionadas de forma completamente aleatoria. Por ello, cabría plantearse en futuros estudios el usar este tipo de selección muestral, así como comprobar la validez de contenido del cuestionario
(Delgado-Rico, Carretero-Dios y Ruch, 2012). A pesar de ello, al establecer que la versión breve puede ser empleada para el análisis de la ansiedad rasgo y estado en universitarios, se aporta una nueva evidencia de su correcto funcionamiento en población general. Este hallazgo puede tener grandes aplicaciones favoreciendo la investigación y la evaluación de este colectivo, con un menor número de ítems, pero una fiabilidad similar a la de la versión completa.

\section{Referencias}

Antúnez, Z., y Vinet, E. V. (2012). Escalas de depresión, ansiedad y Estrés (DASS-21): Validación de la Versión abreviada en Estudiantes Universitarios Chilenos. Terapia Psicológica, 30, 49-55.

Beutel, M. E., Bleichner, F., von Heymann, F., Tritt, K., y Hardt, J. (2011). Inpatient psychosomatic treatment of anxiety disorders: Comorbidities, predictors, and outcomes. International Journal of Clinical and Health Psychology, 11, 443-457.

Brown, T. A. (2006). Data Issues in CFA: Missing, Non-Normal, and Categorical Data. En Kenny, D.A. (Ed.), Confirmatory Factor Analysis for Applied Research (pp. 363-411). New York: The Guilford Press.

Buela-Casal, G., y Guillén-Riquelme, A. (En prensa). Short version of Spanish adaptation of the State-Trait Anxiety Inventory.

Buela-Casal, G., Guillén-Riquelme, A., y Seisdedos, N. (2011). STAI: Cuestionario de Ansiedad Estado-Rasgo. Adaptación española ( $8^{\mathrm{a}}$ ed.). Madrid: TEA Ediciones.

Delgado-Rico, E., Carretero-Dios, H., y Ruch, W. (2012). Content validity evidences in test development: An applied perspective. International Journal of Clinical and Health Psychology, 12, 449-460.

Fioravanti-Bastos, A. C. M., Cheniaux, E., y Landeira-Fernandez, J. (2011). Development and Validation of a Short-Form Version of the Brazilian State-Trait Anxiety Inventory. Psicologia: Reflexão e Critica, 24, 485-494.

Guillén-Riquelme, A., y Buela-Casal, A. (2011). Actualización psicométrica y funcionamiento diferencial de los ítems en el State Trait Anxiety Inventory (STAI). Psicothema, 23, 510-515.

Hartley, J. (2012). New ways of making academic articles easier to read. International Journal of Clinical and Health Psychology, 12, 143-160.

Kaipper, M. B., Chachamovich, E., Hidalgo, M. P. L., Torres, I. L. S., y Caumo, W. (2010). Evaluation of the structures of Brazilian State-Trait Anxiety Inventory using a Rasch psychometric approach. Journal of Psychosomatic Research, 68, 223-233.

Kämpfe, C. K., Gloster, A. T., Wittchen, H. U., Helbig-Lang, S., Lang, T., ... Gerlach, A. L. (2012). Experiential avoidance and anxiety sensitivity in patients with panic disorder and agoraphobia: Do both constructs measure the same? International Journal of Clinical and Health Psychology, 12, 5-22.

Martínez-López, P., Durá Ferrandis, E., Andreu Vaillo, Y., Galón Garrido, M. J., Murgui Pérez, S., y Ibáñez Guerra, E. (2012). Structural validity and distress screening potential of the Hospital Anxiety and Depression Scale in cancer. International Journal of Clinical and Health Psychology, 12, 435-447.

Marteau, T. M., y Bekker, H. (1992). The development of a six-itm shortform of the state scale of the Spielberger State-Trait Anxiety Inventoy (STAI). British Journal of Clinical Psychology, 31, 301-306.

Micin, S., y Bagladi, V. (2011). Salud Mental en Estudiantes Universitarios: Incidencia de Psicopatología y Antecedentes de Conducta Suicida en Población que Acude a un Servicio de Salud Estudiantil. Terapia Psicológica, 29, 53-64.

Muñiz, J., Elosua, P., y Hambleton, R. K. (2013). Directrices para la traducción y adaptación de los tests: segunda edición. Psicothema, 25, 151-157. 
Muñiz, J., y Fernández-Hermida, J. R. (2010). La opinión de los psicólogos españoles sobre el uso de los test. Papeles del Psicólogo, 31, 108-121.

Perpiñá-Galvañ, J., Richart-Martínez, M., y Cabañero-Martínez, M. J. (2011). Fiabilidad y validez de una versión corta de la escala de medida de la ansiedad STAI en pacientes respiratorios. Archivos de Bronconeumología, 47, 184-189.

Perpiñá-Galvañ, J., Richart-Martínez, M., Cabañero-Martínez, M. J., y Martínez-Durá, I. (2011). Validez de contenido de versión corta de la subescala del Cuestionario State-Trait Anxiety Inventory (STAI). Revista Latino-Americana de Enfermagem, 19, A04:1-A04:6.

Rosseel, Y. (2012). Lavaan: An R Package for Structural Equation Modeling. Journal of Statistical Software, 48, 1-36.

Spielberger, C. D. (1983). Manual for the State-Trait Anxiety Inventory: STAI(Form Y). Palo Alto, CA: Consulting Psychologists Press.

Spielberger, C. D., Gorsuch, R. L., y Lushene, R. E. (1970). Manual for the State-Trait Anxiety Inventory. Palo Alto, California: Consulting Psychologist Press.
Spielberger, C. D., y Reheier, E. C. (2009). Assessment of Emotions: Anxiety, Anger, Depression, and Curiosity. Applied Psychology: Health and Well-Being, 1, 271-302. doi:10.1111/j.1758-0854.2009.01017.x.

van Knippenberg, F. C. E., Duivenvoorden, H. J., Bonke, B., y Passchier, J. (1990). Shortening the State-Trait Anxiety Inventory. Journal of Clinical Epidemiology, 43, 995-1000.

Vera-Villarroel, P., Celis-Atenas, K., Córdova-Rubio, N., Buela-Casal, G., y Spielberger, C. D. (2007). Preliminary Analysis and Normative Data of the State-Trait Anxiety Inventory (STAI) in Adolescent and Adults of Santiago, Chile. Terapia Psicológica, 25, 155-162.

Watson, D., Clark, L. A., y Stasik, S. M. (2011). Emotions and the emotional disorders: A quantitative hierarchical perspective. International Journal of Clinical and Health Psychology, 11, 429-442.

Yik, M., Russell, J., y Feldman-Barrett, L. (1999). Structure of self-reported current affect: integration and beyond. Journal of Personality and Social Psychology, 77, 600-619. 
